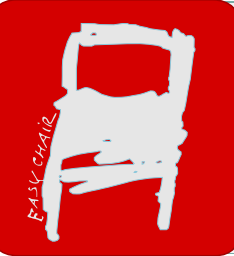

EPiC Series in Health Sciences

EPIC

Health

Volume 2, 2018, Pages 190-192

Sciences

CAOS 2018. The 18th Annual Meeting of the International Society for Computer Assisted Orthopaedic Surgery

\title{
Robot assisted versus Conventional Total Knee Arthroplasty: A prospective randomized controlled trial featuring long term follow-up
}

\author{
Eun-Kyoo Song, Jong-Keun Seon, Won-Gyun Lee \\ Center for Joint Disease, Chonnam National University Bitgoeul Hospital, Gwang-ju, \\ Korea
}

\begin{abstract}
This study determined if robot assisted total knee arthroplasty (TKA) allows for more accurate alignment, better clinical outcome and long term survival rate compared with conventional TKA. 155 consecutive patients who underwent primary TKA were followed more than ten years. Robotic-assisted TKA appears to reduce the number of mechanical axis alignment outliers without any differences in clinical scores, complications and survival rates when compared to conventional manual techniques.
\end{abstract}

Introduction: Well balanced knees with good alignment are essential for a wellfunctioning TKA with long survival of its implants. Robotic TKA is more accurate with less variation in the mechanical axis in spite of no difference in clinical outcomes compared to conventional TKA. No study has compared the long-term follow-up outcomes of robotic versus conventional TKAs using the same implant. The purpose of this prospective comparative study was to compare long term clinical and radiographic outcomes and survival rates for patients that underwent robot assisted TKA or conventional TKA using fixed bearing implants at a mean follow-up of ten years. 
Methods: A randomized controlled study was conducted to compare the clinical and radiographic outcomes of robot assisted and conventional TKA in 155 consecutive patients who underwent primary TKA using fixed bearing implants for knee osteoarthritis from January 2005 to December 2007. Mean follow-up of the patients was 10.3 years in the robot group and 10.1 years in the conventional group. Clinical outcomes were evaluated using range of motion, HSS and WOMAC scores. Radiographic outcomes were assessed using mechanical axis, coronal and sagittal alignments. Radiolucency, perioperative complications and revision rate were also evaluated.

Results: We found no significant differences in clinical outcome parameters between both groups. Radiological outcomes showed that the robot assisted TKA group had more neutrally positioned femoral components in the coronal and sagittal plane with fewer mechanical axis outliers that were classified patients with more than 3 degree of mechanical axis. (8 cases vs 15 cases, $\mathrm{p}<0.05$ ). Mechanical omplications in conventional TKA group included 1 case of instability, 1 case of aseptic loosening and 1 case of polyethylene wear. In robot assisted TKA group, 1 case of aseptic loosening and 1 case of PE wear were found. 4 cases of femoral, 2 cases of tibial radiolucency were occurred in conventional TKA group, while only 1 case of femoral radiolucency was found in Robot assisted TKA group $(11.8 \%$ vs $1.4 \%, p<0.05)$. In survival rate, 2 cases in robot group and 1 case in conventional group underwent revision surgeries and there was no statistically significant difference. ( $97.3 \%$ vs $98 \%, p=0.78)$.

Conclusions: This study demonstrated that robot assisted TKA showed a more accurate alignment of lower leg and position of the prosthesis compared with those of conventional TKA. However, surgical robot system in TKAs showed similar clinical outcomes compared with conventional TKA at long term follow-up. Survival rate also showed no statistically significant difference between robot assisted and conventional TKA. 
Key words: Total knee arthroplasty, Robot-assisted, Conventional

\section{References}

1. Griffin FM, Insall JN, Scuderi GR. Accuracy of soft tissue balancing in total knee arthroplasty. J arthroplasty. 2000 Dec;15(8):970-973.

2. Takahashi T, Wada Y, Yamamoto H. Soft-tissue balancing with pressure distribution during total knee arthroplasty. J Bone Joint Surg Br. 1997 Mar;79(2):235-239.

3. Jacofsky DJ, Allen M. Robotics in Arthroplasty: A Comprehensive Review. J arthroplasty. 2016 Oct;31(10):2353-2363.

4. Song EK, Seon JK, Yim JH, Netravali NA, Bargar WL. Robotic-assisted TKA reduces postoperative alignment outliers and improves gap balance compared to conventional TKA. Clin Orthop Relat Res. 2013 Jan;471(1):118-126.

5. Hampp EL, Chughtai M, Scholl LY, Sodhi N, Bhowmik-Stoker M, Jacofsky DJ, Mont MA. Robotic-Arm Assisted Total Knee Arthroplasty Demonstrated Greater Accuracy and Precision to Plan Compared with Manual Techniques. J Knee Surg. 2018 May 1. doi: 10.1055/s-0038-1641729. 